\title{
PENENTUAN MATRIKS METZLER UNTUK POLINOMIAL STABIL ORDE DUA
}

\author{
RIO SAPUTRA, ZULAKMAL \\ Program Studi Matematika, \\ Fakultas Matematika dan Ilmu Pengetahuan Alam, Universitas Andalas, \\ Kampus UNAND Limau Manis Padang, Indonesia, \\ riosaputra.riosaputra@ymail.com
}

\begin{abstract}
Abstrak. Pada paper ini dikaji sifat yang menjamin eksistensi matriks Metzler $A$ untuk suatu polinomial stabil $p_{2}(s)$ sedemikian sehingga $p_{2}(s)=\operatorname{det}(s I-A)$.

Kata Kunci: sistem kontrol linier kontinu, matriks Metzler, polinomial stabil
\end{abstract}

\section{Pendahuluan}

Diberikan suatu sistem linier kontinu sebagai berikut :

$$
\dot{\mathbf{x}}(t)=A \mathbf{x}(t)+B \mathbf{u}(t), \mathbf{x}(0)=\mathbf{x}_{0},
$$

dimana $\mathbf{x}(t) \in \mathbb{R}^{n}, \mathbf{u}(t) \in \mathbb{R}^{m}, A \in \mathbb{R}^{n \times n}$ dan $B \in \mathbb{R}^{n \times m}$. Sistem (1.1) dikatakan positif jika $\mathbf{x}(t) \in \mathbb{R}_{+}^{n}$ dengan kondisi awal $\mathbf{x}(0)=x_{0} \in \mathbb{R}_{+}^{n}$ dan setiap input $\mathbf{u}(t) \in \mathbb{R}_{+}^{m}, t \geq 0$ [1]. Sistem (1.1) dikatakan stabil asimtotik jika $\lim _{t \rightarrow \infty} \mathbf{x}(t)=\mathbf{0}$ untuk setiap $\mathbf{x}(0) \in \mathbb{R}_{+}^{n}[2]$.

Dalam Farina dan Rinaldi [2] dinyatakan bahwa (1.1) adalah positif jika dan hanya jika $A \in \mathbb{M}_{n}$ dan $B \in \mathbb{R}_{+}^{n \times m}$. Selain itu juga dinyatakan bahwa jika sistem (1.1) adalah positif, maka ia adalah stabil asimtotik jika dan hanya jika semua koefisien dari polinomial

$$
\begin{aligned}
p_{n}(s) & =\operatorname{det}\left(I_{n} s-A\right) \\
& =s^{n}+a_{n-1} s^{n-1}+\cdots+a_{1} s+a_{0}
\end{aligned}
$$

adalah positif, yaitu $a_{i}>0$ untuk $i=0,1, \cdots, n-1$. Dalam hal ini polinomial $p_{n}(s)$ disebut sebagai polinomial stabil untuk sistem positif (1.1).

\section{Penentuan Matriks Metzler untuk Polinomial Stabil Orde Dua}

Berikut diberikan definisi matriks Metzler.

Definisi 2.1. [4] Matriks $A=\left[a_{i j}\right] \in \mathbb{R}^{n \times n}$ dikatakan matriks Metzler jika entri-entri selain entri diagonalnya adalah nonnegatif, yaitu $a_{i j} \geq 0$ untuk $i \neq$ $j, i, j=1,2, \cdots, n$.

Definisi 2.2. [4] Suatu matriks $A \in \mathbb{R}^{n \times n}$ disebut matriks monomial jika setiap baris dan kolomnya memuat hanya satu entri positif dan entri-entri lainnya bernilai nol. 
Lema 2.3. [4] Invers dari matriks monomial A sama dengan transpose dari matriks A, dimana setiap entri taknol digantikan oleh inversnya.

Lema 2.4. [4] Jika $A_{M} \in \mathbb{M}_{n}$ maka $\bar{A}_{M}:=P A_{M} P^{-} 1 \in \mathbb{M}_{n}$ untuk setiap matriks monomial $P \in \mathbb{R}_{+}^{n \times n}$ dan

$$
\operatorname{det}\left(I_{n} s-\bar{A}_{M}\right)=\operatorname{det}\left(I_{n} s-A_{M}\right)
$$

Bukti. Misal $P \in \mathbb{R}_{+}^{n \times n}$ adalah matriks monomial, maka $P^{-1} \in \mathbb{R}_{+}^{n \times n}$. Jika $A_{M} \in$ $\mathbb{M}_{n}$, maka untuk $P$ tersebut ada matriks $\bar{A}_{M}$ yang similar dengan $A_{M}$, yaitu

$$
\bar{A}_{M}=P A_{M} P^{-1}
$$

Karena $A_{M} \in \mathbb{M}_{n}$, maka $\bar{A}_{M} \in \mathbb{M}_{n}$. Selain itu,

$$
\begin{aligned}
\operatorname{det}\left(I_{n} s-\bar{A}_{M}\right) & =\operatorname{det}\left(I_{n} s-P A_{M} P^{-1}\right) \\
& =\operatorname{det}\left(P\left[I_{n} s-A_{M}\right] P^{-1}\right) \\
& =\operatorname{det}(P) \operatorname{det}\left(I_{n} s-A_{M}\right) \operatorname{det}\left(P^{-1}\right) \\
& =\operatorname{det}\left(I_{n} s-A_{M}\right) .
\end{aligned}
$$

Teorema 2.5. [2] Sistem (1.1) adalah stabil asimtotik jika dan hanya jika semua koefisien dari polinomial

$$
\begin{aligned}
p_{n}(s) & =\operatorname{det}\left(I_{n} s-A\right) \\
& =s^{n}+a_{n-1} s^{n-1}+\cdots+a_{1} s+a_{0}
\end{aligned}
$$

adalah positif, yaitu $a_{i}>0$ untuk $i=0,1, \cdots, n-1$.

Lema 2.6. [5] Semua nilai eigen dari matriks Metzler

$$
A_{M}=\left[\begin{array}{cc}
-a_{11} & a_{12} \\
a_{21} & -a_{22}
\end{array}\right], \quad a_{i j} \geq 0, i, j=1,2,
$$

adalah riil.

Bukti. Perhatikan bahwa

$$
\begin{aligned}
\operatorname{det}\left(s I-A_{M}\right) & =\left|\begin{array}{cc}
s+a_{11} & -a_{12} \\
-a_{21} & s+a_{22}
\end{array}\right| \\
& =\left(s+a_{11}\right)\left(s+a_{22}\right)-a_{12} a_{21} \\
& =s^{2}+\left(a_{11}+a_{22}\right) s+a_{11} a_{22}-a_{12} a_{21} .
\end{aligned}
$$

Sehingga nilai eigen dari $A_{M}$ adalah

$$
s_{1,2}=\frac{-\left(a_{11}+a_{22}\right) \pm \sqrt{\left(a_{11}+a_{22}\right)^{2}-4\left(a_{11} a_{22}-a_{12} a_{21}\right)}}{2} .
$$

Dari (2.4) diperoleh bahwa $s_{1,2}$ adalah riil jika dan hanya jika

$$
\left(a_{11}+a_{22}\right)^{2} \geq 4\left(a_{11} a_{22}-a_{12} a_{21}\right) .
$$


24 Rio Saputra, Zulakmal

Penjabaran dari (2.5) yaitu

$$
\begin{aligned}
a_{11}^{2}+a_{22}^{2}+2 a_{11} a_{22} & \geq 4 a_{11} a_{22}-4 a_{12} a_{21} \\
a_{11}^{2}+a_{22}^{2}-2 a_{11} a_{22}+4 a_{12} a_{21} & \geq 0 \\
\left(a_{11}-a_{22}\right)^{2}+4 a_{12} a_{21} & \geq 0
\end{aligned}
$$

yang adalah benar. Jadi semua nilai eigen dari $A_{M}$ adalah riil.

Lema berikut memberikan syarat yang menjamin eksistensi matriks Metzler $A$ untuk polinomial stabil orde dua.

Lema 2.7. [5] Untuk suatu polinomial stabil

$$
p_{2}(s)=s^{2}+a_{1} s+a_{0},
$$

terdapat suatu matriks Metzler $A_{M}$ dengan entri diagonal

$$
\begin{aligned}
& a_{11}=\frac{1}{2}\left(a_{1} \pm \sqrt{a_{1}^{2}-4\left(a_{0}+a_{12} a_{21}\right)}\right), \\
& a_{22}=\frac{1}{2}\left(a_{1} \mp \sqrt{a_{1}^{2}-4\left(a_{0}+a_{12} a_{21}\right)}\right)
\end{aligned}
$$

dan $a_{12} \geq 0, a_{21} \geq 0$ yang memenuhi sifat

$$
a_{1}^{2}-4\left(a_{0}+a_{12} a_{21}\right) \geq 0
$$

jika dan hanya jika

$$
4 a_{0} \leq a_{1}^{2}
$$

Bukti. $(\Rightarrow)$ Polinomial karakteristik dari $A_{M}$ adalah

$$
\begin{aligned}
p_{2}(s) & =\operatorname{det}\left(s I-A_{M}\right) \\
& =\left|\begin{array}{cc}
s+a_{11} & -a_{12} \\
-a_{21} & s+a_{22}
\end{array}\right| \\
& =\left|\begin{array}{cc}
s+\frac{1}{2}\left(a_{1} \pm \sqrt{a_{1}^{2}-4\left(a_{0}+a_{12} a_{21}\right)}\right) & -a_{12} \\
-a_{21} & s+\frac{1}{2}\left(a_{1} \mp \sqrt{a_{1}^{2}-4\left(a_{0}+a_{12} a_{21}\right)}\right)
\end{array}\right| \\
& =s^{2}+\left(a_{11}+a_{22}\right) s+a_{11} a_{22}-a_{12} a_{21} .
\end{aligned}
$$

Sehingga nilai eigen dari $A_{M}$ adalah

$$
s_{1,2}=\frac{-\left(a_{11}+a_{22}\right) \pm \sqrt{\left(a_{11}+a_{22}\right)^{2}-4\left(a_{11} a_{22}-a_{12} a_{21}\right)}}{2} .
$$


Karena $A_{M}$ adalah matriks Metzler, maka $s_{1,2}$ adalah riil. Akibatnya

$$
\begin{aligned}
0 \leq & \left(a_{11}+a_{22}\right)^{2}-4\left(a_{11} a_{22}-a_{12} a_{21}\right) \\
= & a_{11}^{2}+a_{22}^{2}-2 a_{11} a_{22}+4 a_{12} a_{21} \\
= & \left(a_{11}-a_{22}\right)^{2}+4 a_{12} a_{21} \\
= & {\left[\frac{1}{2}\left(a_{1} \pm \sqrt{a_{1}^{2}-4\left(a_{0}+a_{12} a_{21}\right)}\right)-\frac{1}{2}\left(a_{1} \mp \sqrt{a_{1}^{2}-4\left(a_{0}+a_{12} a_{21}\right)}\right)\right]^{2} } \\
& +4 a_{12} a_{21} \\
= & \left(\sqrt{a_{1}^{2}-4\left(a_{0}+a_{12} a_{21}\right)}\right)^{2}+4 a_{12} a_{21} \\
= & a_{1}^{2}-4\left(a_{0}+a_{12} a_{21}\right)+4 a_{12} a_{21} \\
= & a_{1}^{2}-4 a_{0} .
\end{aligned}
$$

Jadi

$$
4 a_{0} \leq a_{1}^{2}
$$

$(\Leftarrow)$ Sebaliknya, untuk polinomial stabil (2.6) misalkan $4 a_{0} \leq a_{1}^{2}$. Maka

$$
\begin{aligned}
0 \leq & a_{1}^{2}-4 a_{0} \\
= & a_{1}^{2}-4\left(a_{0}+a_{12} a_{21}\right)+4 a_{12} a_{21} \\
= & \left(\sqrt{a_{1}^{2}-4\left(a_{0}+a_{12} a_{21}\right)}\right)^{2}+4 a_{12} a_{21} \\
= & {\left[\frac{1}{2}\left(a_{1} \pm \sqrt{a_{1}^{2}-4\left(a_{0}+a_{12} a_{21}\right)}\right)-\frac{1}{2}\left(a_{1} \mp \sqrt{a_{1}^{2}-4\left(a_{0}+a_{12} a_{21}\right)}\right)\right]^{2} } \\
& +4 a_{12} a_{21} .
\end{aligned}
$$

Jika $a_{11}$ dan $a_{22}$ seperti yang diperlihatkan dalam (2.7) dan (2.8), maka (2.12) dapat ditulis menjadi

$$
\begin{aligned}
0 & \leq\left(a_{11}-a_{22}\right)^{2}+4 a_{12} a_{21} \\
-4 a_{12} a_{21} & \leq a_{11}^{2}+a_{22}^{2}-2 a_{11} a_{22} \\
4 a_{11} a_{22}-4 a_{12} a_{21} & \leq a_{11}^{2}+a_{22}^{2}+2 a_{11} a_{22} \\
4\left(a_{11} a_{22}-a_{12} a_{21}\right) & \leq\left(a_{11}+a_{22}\right)^{2} \\
0 & \leq\left(a_{11}+a_{22}\right)^{2}-4\left(a_{11} a_{22}-a_{12} a_{21}\right) \\
& =a_{1}^{2}-4 a_{0},
\end{aligned}
$$

dimana

$$
a_{1}=a_{11}+a_{22} \text { dan } a_{0}=a_{11} a_{22}-a_{12} a_{21} .
$$

Karena (2.13) berlaku, maka akar dari $p_{2}(s)$ adalah riil. Akibatnya matriks Metzler $A_{M}$ dengan bentuk (2.2) ada dengan $a_{11}$ dan $a_{22}$ diperlihatkan dalam (2.7) dan (2.8).

Teorema 2.8. [5] Untuk suatu polinomial stabil (2.6), terdapat suatu matriks Metzler $\bar{A}_{M}=P A_{M} P^{-1}$, dimana $P \in \mathbb{R}_{+}^{2 \times 2}$ adalah suatu matriks monomial dan $A_{M}$ 
berbentuk salah satu dari yang berikut.

$$
\begin{aligned}
& A_{M 1}=\left[\begin{array}{cc}
-a & a_{1} a-a^{2}-a_{0} \\
1 & a-a_{1}
\end{array}\right], \\
& A_{M 2}=\left[\begin{array}{cc}
-a & 1 \\
a_{1} a-a^{2}-a_{0} a-a_{1}
\end{array}\right],
\end{aligned}
$$

$0<a<a_{1}, a_{1} a-a^{2}-a_{0} \geq 0$ jika dan hanya jika $4 a_{0} \leq a_{1}^{2}$.

Bukti. $(\Rightarrow)$ Jika $A_{M}=A_{M 1}$, maka dengan memperhatikan (2.2) diperoleh

$$
a_{11}=a, a_{21}=1, a_{12}=a_{1} a-a^{2}-a_{0} \text { dan } a_{22}=a_{1}-a .
$$

Polinomial karakteristik dari $A_{M 1}$ adalah

$$
\begin{aligned}
\operatorname{det}\left(s I-A_{M 1}\right) & =\left|\begin{array}{cc}
s+a & a_{0}+a^{2}-a_{1} a \\
-1 & s+a_{1}-a
\end{array}\right| \\
& =(s+a)\left(s+a_{1}-a\right)+a_{0}+a^{2}-a_{1} a \\
& =s^{2}+a_{1} s+a_{0} .
\end{aligned}
$$

Jika $A_{M}=A_{M 2}$, maka dengan memperhatikan (2.2) diperoleh

$$
a_{11}=a, a_{12}=1, a_{21}=a_{1} a-a^{2}-a_{0} \operatorname{dan} a_{22}=a_{1}-a .
$$

Polinomial karakteristik dari $A_{M 2}$ adalah

$$
\begin{aligned}
\operatorname{det}\left(s I-A_{M 2}\right) & =\left|\begin{array}{cc}
s+a & -1 \\
a_{0}+a^{2}-a_{1} a s+a_{1}-a
\end{array}\right| \\
& =(s+a)\left(s+a_{1}-a\right)+a_{0}+a^{2}-a_{1} a \\
& =s^{2}+a_{1} s+a_{0} .
\end{aligned}
$$

Karena $A_{M}$ adalah matriks Metzler, maka $\bar{A}_{M}$ juga matriks Metzler (berdasarkan Lema 2.4), sehinggga nilai eigen dari $\bar{A}_{M}$ adalah riil. Akibatnya

$$
4 a_{0} \leq a_{1}^{2}
$$

$(\Leftarrow)$ Misal $(2.6)$ adalah polinomial stabil yang bersifat $4 a_{0} \leq a_{1}^{2}$. Berdasarkan Lema 2.7 terdapat matriks Metzler

$$
A_{M}=\left[\begin{array}{cc}
-a_{11} & a_{12} \\
a_{21} & -a_{22}
\end{array}\right]
$$

dengan entri diagonal (2.7) dan (2.8), dan $a_{12} \geq 0, a_{21} \geq 0$ memenuhi (2.9). Misal $a_{11}=a, a_{21}=1, a_{12}=a_{1} a-a^{2}-a_{0}$ dan $a_{22}=a_{1}-a$ maka diperoleh

$$
A_{M 1}=\left[\begin{array}{cc}
-a & a_{1} a-a^{2}-a_{0} \\
1 & a-a_{1}
\end{array}\right] \text {, }
$$

Selanjutnya, jika $a_{11}=a, a_{12}=1, a_{21}=a_{1} a-a^{2}-a_{0}$ dan $a_{22}=a_{1}-a$, maka diperoleh

$$
A_{M 2}=\left[\begin{array}{cc}
-a & 1 \\
a_{1} a-a^{2}-a_{0} & a-a_{1}
\end{array}\right] .
$$


Dalam kedua kasus diperoleh

$$
\bar{A}_{M}=P A_{M} P^{-1}
$$

yang adalah matriks Metzler untuk suatu matriks monomial $P \in \mathbb{R}_{+}^{2 \times 2}$.

Contoh 2.9. Tentukan matriks Metzler yang sesuai untuk polinomial stabil

$$
p_{2}(s)=s^{2}+6 s+8 .
$$

Polinomial (2.17) memenuhi kondisi (2.10) karena $a_{1}^{2}=36$ dan $4 a_{0}=32$. Akar-akar dari (2.17) adalah $s_{1}=-4, s_{2}=-2$. Matriks Metzler yang sesuai untuk (2.17) adalah

$$
\bar{A}_{M 1}=P A_{M 1} P^{-1} \text { atau } \bar{A}_{M 2}=P A_{M 2} P^{-1}
$$

dimana

$$
A_{M 1}=\left[\begin{array}{cc}
-a & 6 a-a^{2}-8 \\
1 & a-6
\end{array}\right], A_{M 2}=\left[\begin{array}{cc}
-a & 1 \\
6 a-a^{2}-8 & a-6
\end{array}\right]
$$

dan untuk suatu matriks monomial $P \in \mathbb{R}_{+}^{2 \times 2}$, dengan $2 \leq a \leq 4$.

Pilih matriks monomial

$$
P=\left[\begin{array}{ll}
0 & 2 \\
4 & 0
\end{array}\right]
$$

Selanjutnya dengan menggunakan (2.18) dan (2.19), diperoleh himpunan matriks Metzler yang memenuhi (2.17) adalah

$$
\begin{aligned}
& \bar{A}_{M 1}=P A_{M 1} P^{-1} \\
& =\left[\begin{array}{ll}
0 & 2 \\
4 & 0
\end{array}\right]\left[\begin{array}{cc}
-a & 6 a-a^{2}-8 \\
1 & a-6
\end{array}\right]\left[\begin{array}{cc}
0 & \frac{1}{4} \\
\frac{1}{2} & 0
\end{array}\right] \\
& =\left[\begin{array}{cc}
a-6 & \frac{1}{2} \\
\frac{1}{2}\left(24 a-4 a^{2}-32\right) & -a
\end{array}\right] \\
& \text { atau } \\
& \bar{A}_{M 2}=P A_{M 2} P^{-1} \\
& =\left[\begin{array}{ll}
0 & 2 \\
4 & 0
\end{array}\right]\left[\begin{array}{cc}
-a & 1 \\
6 a-a^{2}-8 & a-0
\end{array}\right]\left[\begin{array}{cc}
0 & \frac{1}{4} \\
\frac{1}{2} & 0
\end{array}\right] \\
& =\left[\begin{array}{cc}
a-6 & \frac{1}{4}(12 a-2 a-16) \\
2 & -a
\end{array}\right] \text {. }
\end{aligned}
$$

\section{Penutup}

Jika diberikan polinomial stabil $p_{2}(s)=s^{2}+a_{1} s+a_{0}$, maka syarat yang menjamin eksistensi matriks Metzler $A$ sedemikian sehingga

$$
\operatorname{det}(s I-A)=p_{2}(s)
$$

adalah $4 a_{0} \leq a_{1}^{2}$ 
28 Rio Saputra, Zulakmal

\section{Ucapan Terima kasih}

Penulis mengucapkan terima kasih kepada Bapak Dr. Muhafzan, Bapak Dr. Dodi Devianto, Bapak Dr. Admi Nazra, dan Bapak Efendi, M.Si yang telah memberikan masukan dan saran sehingga paper ini dapat diselesaikan dengan baik.

\section{Daftar Pustaka}

[1] Anton, H. 1991. Aljabar Linier Elementer. Edisi Kedelapan, Jilid-1. Erlangga, Jakarta

[2] Farina, L. dan Rinaldi, S. 2000. Positive Linear System Theory and Applications. J. Wiley. New York

[3] Kaczorek, T. 1992. Linear Control System. Vol. 1, Research Studies Press. J. Wiley. New York

[4] Kaczorek, T. 2002. Positive 1D and 2D System. Springer Verlag. London

[5] Kaczorek, T. 2012. Existence and determination of the Set of Metzler Matrices for Given Polynomials. International Journal of Applied Mathematics and Computer Sience 22(2): $349-398$ 